\title{
DETERMINACIÓN DE CALIDAD HÍDRICA EN SISTEMAS DE PRODUCCIÓN PORCINA CON CERO VERTIMIENTOS, EN RESERVA FORESTAL DE LOS CERROS ORIENTALES DE BOGOTÁ, D.C.
}

\author{
HYDRIC QUALITY DETERMINATION IN SWINE \\ PRODUCTION SYSTEMS WITHOUT WASTEWATERS \\ IN THE EASTERN OF FOREST RESERVE IN BOGOTA
}

\author{
Luz Elena Santacoloma V., ${ }^{1}$ Carmen Rosa Montes P., ${ }^{2}$ Jorge Rondón ${ }^{3}$
}

\begin{abstract}
RESUMEN
La actividad porcina desarrollada en un ambiente de fragilidad eco sistémica, como el área de Reserva de los Cerros Orientales de Bogotá, ubicada entre 3.000 y 3.200 msnm, exige que se contemplen modelos tecnológicos que garanticen la permanencia de bienes y servicios ambientales, la sostenibilidad económica de los habitantes de la región y el bienestar de la población. Se evaluó el programa "cero vertimientos" en 7 fincas de producción porcina, localizadas sobre las márgenes de los afluentes del río Teusacá a $3.200 \mathrm{msnm}$, en la vereda el Verjón alto, de la ciudad de Bogotá. Se tomaron muestras puntuales de agua superficial en cada predio antes y después de la unidad de producción. En campo se obtuvo el pH, temperatura y oxigeno disuelto. En laboratorio se determinaron amonio, coliformes fecales, coliformes totales, DBO, DQO, fósforo total, grasa y aceites, nitrógeno total Kjeldahl, sólidos suspendidos totales y sólidos sedimentables. Se realizaron análisis de los resultados comparándolos con la normatividad vigente. Los parámetros físicos y químicos presentaron valores dentro de los límites permisibles. A partir de lo anterior, se pudo establecer que el agua de la región en estudio tiene alta calidad en función de los propósitos de la reserva.
\end{abstract}

Palabras clave: calidad hídrica, contaminación hídrica, cero vertimientos, porcinos

\footnotetext{
1 Zootecnista, Magister en Gestión ambiental para el desarrollo sostenible, docente Escuela de Ciencias Agrícolas, Pecuarias y del Medio Ambiente. Universidad Nacional Abierta y a Distancia, UNAD. Bogotá, Colombia, luz. santacoloma@unad.edu.co.

2 Ingeniera Forestal, DEA Economie de 1'environnement et des ressources naturelles (Paris, France), docente Escuela de Ciencias Agrícolas, Pecuarias y del Medio Ambiente. Universidad Nacional Abierta y a Distancia, UNAD. Bogotá, Colombia,. carmen.montes@unad.edu.co.

3 Ingeniero de Alimentos, docente Escuela de la Escuela de Ciencias Básicas Tecnología e Ingeniería. Universidad Nacional Abierta y a Distancia, UNAD. Bogotá, Colombia. jorge.rondon@unad.edu.co
} 


\begin{abstract}
Swine activity developed in an environment of fragile ecosystems, such as reserve area the eastern hills of Bogota, located between 3,000 and 3,200 meters, requires contemplated technological models that guarantee the permanence of environmental goods and services, economic sustainability the habitants of the region and population welfare. We evaluated the program • zero effluents "in 7 swine production farms, located on the banks of the tributaries of the river Teusacá to 3,200 m of the "Verjón Alto", village of Bogotá, DC. Samples were taken off of surface water in each site before and after the production unit. It was obtained in situ $\mathrm{pH}$, temperature and dissolved oxygen. Ammonium were determined in laboratory, fecal coliforms, total coliforms, BOD, COD, total phosphorus, fat and oils, total Kjeldahl nitrogen, total suspended solids and settleable solids. Analysis was carried out by comparing results with regulations. The physical and chemical values were within permissible limits. In short the water in the study area has high quality in terms of the purposes of the reservation.
\end{abstract}

Key words: swine, water pollution, water quality.

Aprobado: Junio 29 de 2010

\title{
INTRODUCCIÓN
}

Los Sistemas Productivos Porcinos son considerados de alto impacto ambiental sobre suelo, atmósfera y, especialmente, sobre fuentes de agua superficiales y subterráneas, ya que generan residuos por limpieza de instalaciones, sobrantes de alimentos y excretas de los animales (heces y orina); además, el $82 \%$ del agua que ingresa a las granjas sale como agua residual, acompañada de heces, orina, alimento desperdiciado y otros materiales que se arrastran a través de los drenajes (Bravo, 2008). De otra parte, una unidad productiva de 100 hembras genera una carga orgánica de sólidos suspendidos totales (SST) de 23.013 $\mathrm{mg} / \mathrm{L}$ y una demanda bioquímica de oxígeno (DBO) de $7.239 \mathrm{mg} / \mathrm{L}$, parámetros que superan los establecidos por las normas ambientales vigentes y aplicadas en Colombia (Bravo, 2008).

De acuerdo con lo anterior, Piccinini (1995) plantea que en las características de las aguas residuales porcinas y del volumen final por evacuar influyen factores como la alimentación, el estado fisiológico del animal, el tipo de estabulación y el sistema de limpieza, entre otros. El mismo autor cita una producción diaria de agua residual (sin dilución de agua usada para limpieza del sitio) por cabeza de $1.4 \mathrm{~kg} /$ día para lechones en crecimiento de $25 \mathrm{~kg}$ y de 5,2 $\mathrm{kg} /$ día para un cerdo de engorde de $80 \mathrm{~kg}$. Refiere también información indicando que para limpieza "en seco" se genera una producción de agua residual de 3,5 metros cúbicos por quintal $^{4}$ de peso vivo y un valor medio en el agua residual de 4,8 kg/ton de nitrógeno, 1,6 $\mathrm{kg} /$ ton de fósforo y $3,1 \mathrm{~kg} / \mathrm{ton}$ de potasio para el piso vivo referido.

\footnotetext{
4 Un quintal equivale a $50 \mathrm{~kg}$.
} 
Así mismo, Cubillos (1978) identificó rangos de concentraciones para diferentes parámetros, procedentes de composición de desechos de lavados de pisos en instalaciones de porcinos: DBO: 495-12918, DQO: 1006-16.125, Nitrógeno orgánico: 60-308, Nitrógeno amoniacal: 167-1335, sólidos sedimentables: 20,8-150 mg/l, sólidos totales: 3.850- 12.472, pH: 6-7.9. El mismo autor afirma que los valores varían con la edad, etapa de desarrollo, la alimentación y la cantidad de agua utilizada para el lavado de pisos.

Taiganides et al. (1996), citado por Pérez (2006) encontró en las excretas de porcinos, una relación de tres a uno entre la cantidad de sólidos suspendidos totales (SST) y la demanda bioquímica de oxígeno (DBO). Este autor precisa que un cerdo promedio de 54 kilogramos excreta 331 gramos día.

La actividad porcina desarrollada en un ambiente de fragilidad eco sistémico, como es el área de Reserva de los Cerros Orientales de Bogotá, ubicada entre 3.000 y 3.200 msnm, cuyo objetivo es el mantenimiento de las condiciones ambientales naturales que permitan preservar el equilibrio auto regulador para asegurar su disponibilidad en cantidad y calidad adecuada, exige que se contemplen modelos tecnológicos que garanticen la permanencia de bienes y servicios ambientales, la sostenibilidad económica de los habitantes de la región y el bienestar de la población.

La Corporación Autónoma Regional de Cundinamarca (CAR) adelantó en la vereda el Verjón Alto, área rural del Distrito capital, un programa de "cero vertimientos" en las fincas con sistemas de producción porcina, el cual contempló el cierre de puntos de salida y descarga de vertimientos, el barrido en seco de los residuos de la producción porcina, el acondicionamiento de instalaciones, el almacenamiento y la construcción de composteras. También se consideró el mejoramiento de condiciones sanitarias de la producción para eliminación de focos de infección y proliferación de vectores (CAR, 2006).

La calidad del agua es una variable fundamental del sistema hídrico, tanto en su caracterización ambiental, como desde la perspectiva de la planificación hidrológica. Por tanto, se concibe la calidad del agua como el conjunto de características físicas, químicas y biológicas que hacen que esta sea apropiada para un uso determinado. En la normatividad colombiana esta definición está asociada a la utilización que se le dé al recurso: consumo humano y doméstico, preservación de flora y fauna, agrícola, pecuario, recreativo, industrial y transporte.

En Colombia, en el Decreto 1594 de 1984 se encuentra la norma que regula los usos del agua (anteriormente mencionados) y los residuos líquidos. Allí se establecen, de manera general para el país, algunos criterios de calidad de agua en factores físico-químicos y microbiológicos. Otras normas importantes que se toman como punto de referencia son: el Acuerdo CAR 043 de 2006, el cual establece objetivos de calidad de agua para la Cuenca del rio Bogotá, por lograr en el 2020 y la Resolución 5731 de 2008 de la Secretaría del Ambiente de Bogotá. 
Por lo tanto, el objetivo de este estudio se centró en la evaluación de la eficacia del programa de cero vertimientos, de los sistemas de producción porcina, respecto al cumplimiento de la normatividad hídrica, para mitigar el impacto negativo en las aguas superficiales de siete fincas de la zona de reserva forestal de la vereda el Verjón alto -área rural del Distrito capital.

\section{METODOLOGÍA (MATERIALES Y MÉTODOS)}

\section{Área de estudio}

Se encuentra ubicada en los cerros orientales de Bogotá, área rural, región central de los Andes orientales de Colombia, como se puede observar en el Gráfico 1. El proyecto se desarrolló en siete fincas de la vereda el Verjón alto, localizadas sobre las márgenes de los afluentes del río Teusacá a 3200 m.s.n.m, donde predominan condiciones ambientales propias del subpáramo y precipitación media anual entre 1.000 y $1400 \mathrm{~mm}$., régimen de distribución de lluvias bimodal y períodos de mayor pluviosidad de marzo a mayo y de octubre a noviembre, con períodos secos de diciembre a febrero y de junio a septiembre. La temperatura media anual varía entre 6.0 y 12 grados centígrados. Se presenta nubosidad alta y vientos fuertes. (López et al 1998).

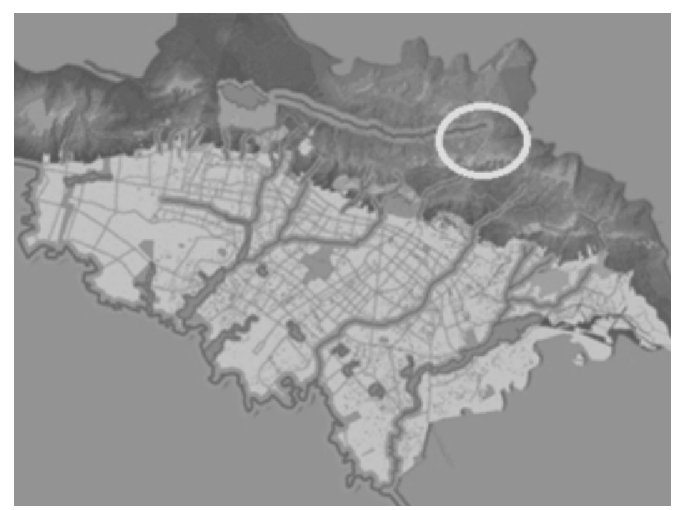

Grafico 1. Localización del estudio en la Reserva de los Cerros Orientales de Bogotá Fuente: http://www.todoarquitectura.com/v2/noticias/one_news.asp?IDNews=2015.

Entre la vegetación dominante se encuentran áreas intervenidas con gramíneas como la Falsa Poa para uso ganadero, cultivos de papa y arveja y matorrales conformados por Weinmania tomentosa, Clusia multiflora, Myrsine guianensis, Bucquetia glutinosa, Clethra fimbriata, Pentacallia pulchellus, Chusquea tessellata y Espeletia sp (CAR y UN 2004), (Camargo et al 2000).

\section{Muestreo}

Para determinar el tamaño de la muestra, se realizó un muestreo estratificado, con una población de 42 fincas, que cumplieran dos requisitos: el primero, que el sistema productivo 
se localizara a un máximo de 100 metros de distancia, respecto a la fuente de agua superficial y, el segundo, que tuviera un número superior a 100 cerdos de levante y ceba. Se determinó el tamaño de la muestra en 7 unidades productivas, con registro de datos antes y después del sistema productivo, para un total de 14 muestras con el fin de identificar una posible contaminación difusa.

Para la recolección de muestras en las fincas, el laboratorio proporcionó los frascos de vidrio, kit de oxigeno disuelto, $\mathrm{pH}$ metro y termómetro. En el campo, se tomaron muestras puntuales en un balde, previamente purgado, sumergiéndolo en la fuente de agua superficial, las cuales, inmediatamente, se transvasaron a cada uno de los frascos. Posteriormente, las muestras se almacenaron en frío en una nevera portátil, para enviarlas el mismo día al laboratorio. En cada predio se tomaron dos muestras: antes y después de la unidad de producción entre las 10 a.m y las 2 p.m para un total de 14 muestras de agua superficial En campo se obtuvo el $\mathrm{pH}$ mediante medidor de $\mathrm{pH}$, temperatura con termómetro y oxigeno disuelto por el método de (Winkler, 1889). Dado que la toma de muestras fue realizada rigurosamente con todos los parámetros técnicos, no se consideró necesario realizar réplicas, ya que una sola muestra por sitio era suficiente.

Para determinar las cantidades de amonio en laboratorio, coliformes fecales, coliformes totales, DBO, DQO, fósforo total, grasa $\mathrm{y}$ aceites, nitrógeno total Kjeldahl, sólidos suspendidos totales y sólidos sedimentables, se utilizaron los métodos colorimétricos $\left(\right.$ Nesslerización $^{5}$ ), filtración por membrana, ${ }^{6-7}$ incubación 5 días (electrométrico ${ }^{8}$ ), reflujo abierto, ${ }^{9}$ colorimétrico (cloruro estañoso ${ }^{10}$ ), extracción Soxhlet, ${ }^{11}$ titulo métrico $\mathrm{H} 2 \mathrm{SO} 4,{ }^{12}$ gravimétrico (secado a 105 grados centígrados).

Como parámetro de calidad se tuvieron en cuenta las directrices dadas por el Decreto 1594 de 1984 del Ministerio de Agricultura para sólidos sedimentables; el Acuerdo CAR 043 de 2006 Clase I para, DBO, amonio, coliformes totales; el Acuerdo Resolución 5731 de 2008 de la Secretaria del Ambiente de Bogotá (2008) para Oxígeno disuelto (tramo 1 canal Torca) DQO, Nitrógeno total, sólidos suspendidos totales, aceites y grasas, $\mathrm{pH}$ y fósforo total; IDEAM (2001) para temperatura y la Norma Oficial Mexicana NOM-003-ECOL1997 para coliformes fecales, como se observa en la Tabla 1.

\footnotetext{
$5 \mathrm{SM} 4500-\mathrm{NH} 3 \mathrm{C}$.

6 SM 9222 D.

7 SM 9222 B.

8 SM 5210 B.

9 SM 5220 B.

10 SM 4500-P D.

11 SM 5520 D.

12 SM 2540 D.
} 
Tabla 1. Referentes normativos utilizados en el estudio

\begin{tabular}{|l|l|}
\hline \multicolumn{1}{|c|}{ Norma } & \multicolumn{1}{c|}{ Parámetro } \\
\hline Decreto 1594 de 1984 & Sólidos sedimentables \\
\hline Acuerdo CAR 043 de 2006 & DBO, amonio y coliformes totales (Clase II) \\
\hline $\begin{array}{l}\text { Resolución 5731 de 2008 de la Secretaria del } \\
\text { Ambiente de Bogotá }\end{array}$ & $\begin{array}{l}\text { Oxigeno disuelto, DQO, Nitrógeno Total, , SST, } \\
\text { Aceites y grasas, pH y Fosforo total }\end{array}$ \\
\hline IDEAM & Temperatura \\
\hline Norma oficial mexicana NOM-003-ECOL-1997 & Coliformes fecales \\
\hline
\end{tabular}

Fuente: los autores 2010

\section{Procesamiento de datos y análisis estadístico}

Teniendo en cuenta el objetivo del estudio, analizar el comportamiento de las variables de calidad de agua superficial de consumo agrícola, respecto a la normatividad vigente nacional e internacional, se aplicaron las siguientes técnicas estadísticas: estadística descriptiva, estadística inferencial, pruebas específicas para hacer comparaciones de medias y de varianzas y prueba $\mathrm{F}$ de Fischer para determinar la homocedasticidad.

Para conocer el comportamiento general de los datos se aplicaron medidas de tendencia central y de dispersión de la información.

Se realizó el análisis de varianza con el fin de conocer la existencia de diferencias significativas entre los factores objeto de estudio, utilizando un nivel de confianza del 95\%. La estadística inferencial se utilizó para determinar si existen diferencias significativas entre los datos de las variables a la entrada y salida de las fincas de dicha región.

Se aplicaron pruebas específicas tales como, LDS (Least significant difference) y la prueba de Dunnet, con el fin de comparar la media del dato experimental con el dato teórico dado por la norma. Para esta prueba se tomó como punto de referencia la media de los puntos de salida de las fincas, ya que el propósito de la investigación era verificar la afectación de la actividad productiva porcina en la calidad del agua.

Los grados de libertad para este proceso es $n-1$, donde n es el tamaño de la muestra; por lo tanto, los grados de libertad para todas las variables analizadas es de 6, excepto para el amonio que es de 4.

La prueba de Fischer para determinar el cumplimiento del principio de homocedasticidad demostró que el uso de la estadística paramétrica es apropiada para este caso. 


\section{Análisis y discusión de resultados}

De acuerdo con los resultados encontrados en el estudio, la actividad porcícola, en el área donde se aplicó el programa de "cero vertimientos", no afecta la calidad del agua superficial de la reserva. Esto se evidencia al comparar los diferentes parámetros químicos, físicos y microbiológicos del agua analizada en las zonas aledañas a las fincas con los parámetros dados por las regulaciones nacionales e internacionales de calidad de agua.

La información obtenida sobre calidad de agua con respecto al parámetro temperatura, se mantuvo constante y no supera $\operatorname{los} 10{ }^{\circ} \mathrm{C}$ para las 14 mediciones realizadas en la región. Lo anterior cumple con lo indicado por el Instituto de Hidrología, Meteorología y Estudios Ambientales -IDEAM- (2001) en cuanto a que este valor tiene coherencia con la temperatura ambiental en la cual se encuentran las fuentes superficiales estudiadas.

La variable $\mathrm{pH}$ presentó el mismo valor en los puntos antes y después del sitio de producción de porcinos para las fincas el Embudo y Hospedaleto, Para Chuscalito y el Regalo se presentaron incrementos de 7,2 a 7,6 y de 7,6 a 7,8, respectivamente, aunque se mantuvieron dentro de los límites permisibles. En la Hondura y los Pinos se obtuvieron valores inferiores a la norma (Resolución 5731 de 2008 de la Secretaria del Ambiente de Bogotá), con una sensible disminución del pH después de que la fuente hídrica pasara por el sistema productivo. Para el Triunfo, este parámetro se incrementó de 5.9 a 6.2, sin hallarse diferencia estadísticamente significativa. Por tanto, se puede afirmar que hay cumplimiento de la norma y se presume que la ligera disminución del $\mathrm{pH}$ en las fincas Los Pinos y la Hondura se debe a las características de acidez del suelo (ver gráfico 2).

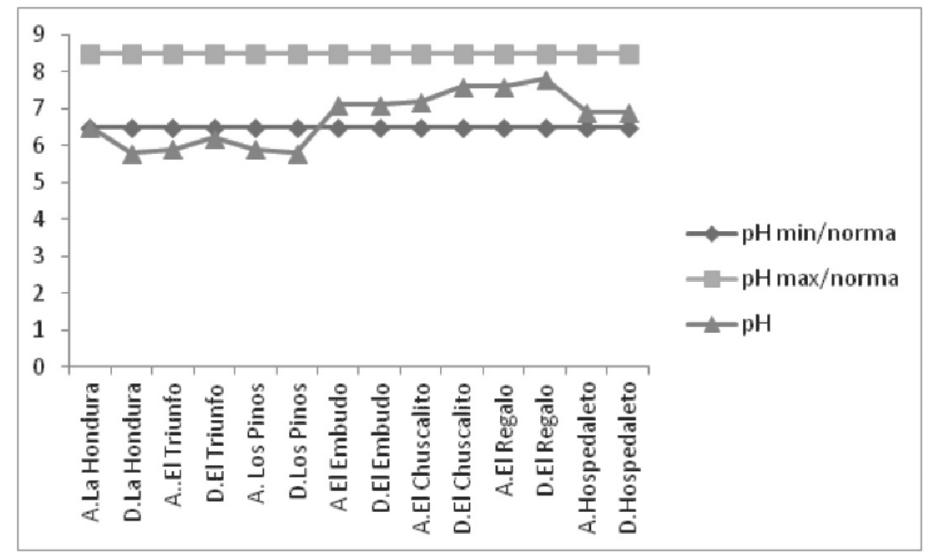

Gráfico 2. Variación de pH en muestras de agua superficial en la vereda Verjón Alto. Bogotá. A: antes del sistema de producción porcino y

D: después del sistema de producción porcina. 
Para el oxígeno disuelto, los resultados de todas las muestras superan favorablemente el valor exigido por la norma del Acuerdo 043 de la CAR y confirman el resultado de calidad esperado en los afluentes de la cuenca alta del río Teusacá donde se realizó la investigación; se cuenta, también, con las características topográficas de alta pendiente que le permiten aireación permanente del agua (gráfico 3).

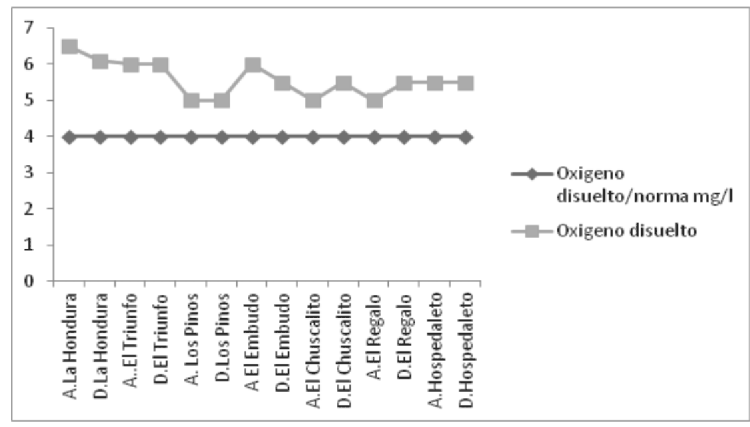

Gráfico 3. Variación de oxigeno disuelto en muestras de agua superficial en la vereda Verjón Alto.

Bogotá. A: antes del sistema de producción porcino y

D: después del sistema de producción porcina.

El amonio registró un valor promedio de $0,042 \mathrm{mg} /$ litro. La concentración más alta se registró en la finca la Hondura en el punto de la fuente hídrica antes de pasar por la unidad productiva, con valor extremo de $0.17 \mathrm{mg} /$ litro. Este valor superó la norma del Acuerdo $043 \mathrm{CAR}$, la cual es de $0.1 \mathrm{mg} / \mathrm{litro}$, presumiblemente por mayor concentración de materia orgánica en el lecho de la quebrada estudiada. Al realizar la prueba estadística de Dunnet, con nivel de confianza del 95\%, se encontró, sin embargo, que en el conjunto de muestras analizadas se cumplen los estándares planteados por la norma. (ver gráfico 4).

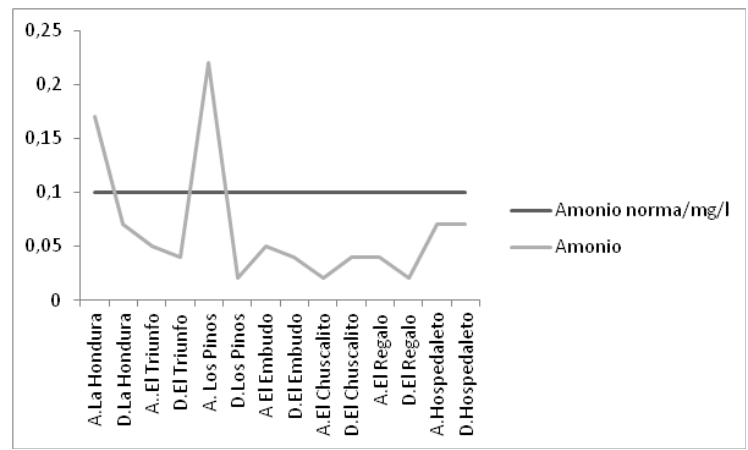

Gráfico 4. Variación de Amonio (mg/l) en muestras de agua superficial en la vereda Verjón Alto.

Bogotá. A: Antes del sistema de producción porcino y

D: Después del sistema de producción porcina. 
Dado que en esta variable las muestras arrojaron dos resultados atípicos, correspondientes a las fincas Hondura y Los Pinos, se consideraron como "datos raros" y no se tuvieron en cuenta para el análisis. Por consiguiente, la prueba de homocedasticidad, se realizó con los resultados que se obtuvieron en las cinco fincas restantes: Triunfo, Embudo, Chuscalito, Regalo y Hospedaleto.

Para los coliformes fecales, en la mayor parte de las muestras analizadas, no hay afectación por parte de los sistemas productivos. Únicamente se encontraron datos por encima de la norma oficial mexicana NOM-003-ECOL-1997 en la finca el Regalo. Al realizarse la prueba de Dunnet unilateral para verificar la significancia del alto valor obtenido en esta finca, se encuentra que está dentro de los parámetros dados por la norma (Ver gráfico 5).

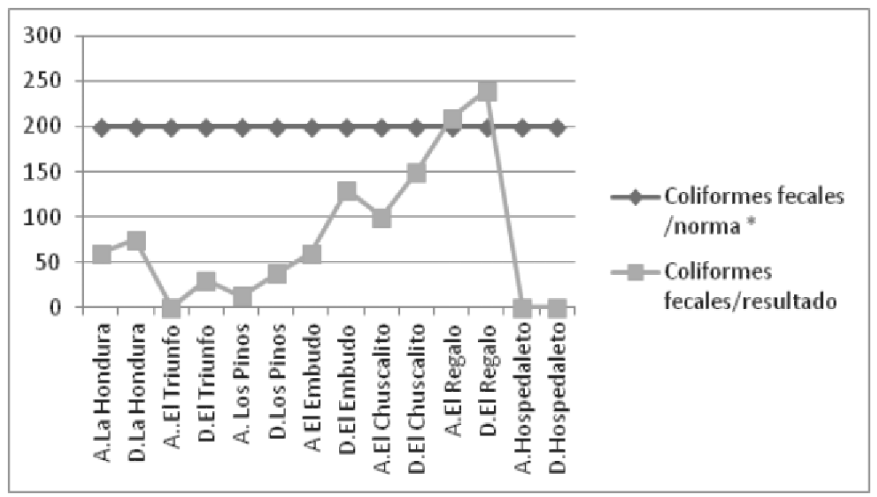

Gráfico 5. Variación de coliformes fecales (UFC/100ml) en muestras de agua superficial en la vereda Verjón Alto. Bogotá. A: Antes del sistema de producción porcino y

D: Después del sistema de producción porcina

Un comportamiento similar presentaron los coliformes totales con un valor de 9800mg/ litro a la entrada y de $4300 \mathrm{UFC} / 100 \mathrm{ml}$ a la salida de la finca el Regalo. El Acuerdo 043 de 2006 de la CAR indica que el valor máximo es de 5000UFC/100L. No obstante, al aplicar la prueba Dunnet unilateral para verificar qué tanto se alejan de la norma estos valores, se obtiene como resultado que el conjunto de aguas de la región está por debajo del límite permisible y que la calidad del agua es aceptable (ver gráfico 6). 


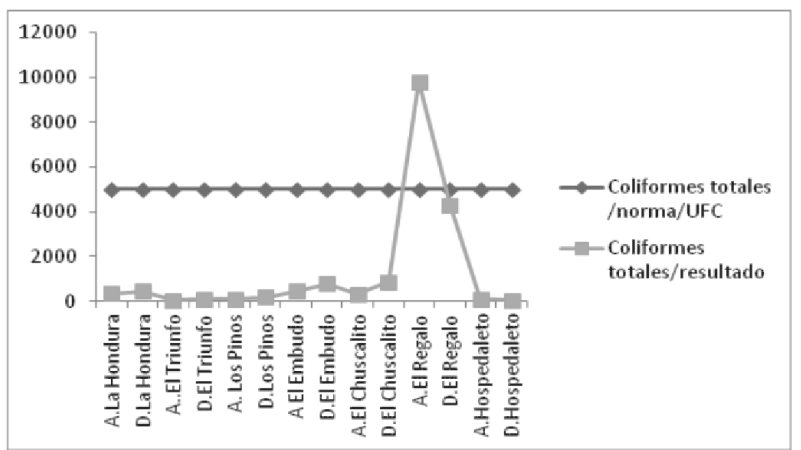

Gráfico 6. Variación de coliformes totales (UFC/100ml) en muestras de agua superficial en la vereda Verjón Alto. Bogotá. A: Antes del sistema de producción porcino y

D: Después del sistema de producción porcina

La demanda Bioquímica de Oxígeno (DBO) fue de $2 \mathrm{mg} /$ litro y resultó constante para todas las muestras realizadas. Este valor está por debajo de lo exigido en el Acuerdo 43 de 2006, que define un máximo para este parámetro de 7 mg/litro (ver gráfico 7).

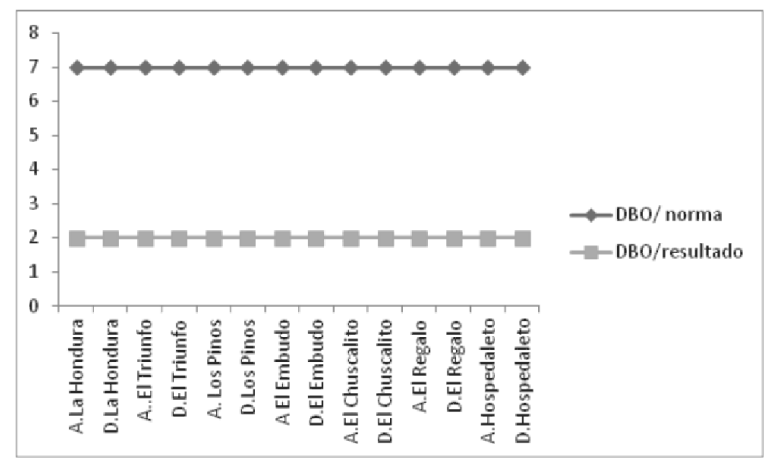

Gráfico 7. Variación de Demanda bioquímica de oxígeno $(\mathrm{mg} / \mathrm{l})$ en muestras de agua superficial en la vereda Verjón Alto. Bogotá. A: Antes del sistema de producción porcino y D: Después del sistema de producción porcina

La demanda química de oxígeno (DQO) presentó un comportamiento similar en todos los casos analizados, con valores por debajo de los estipulados en la Resolución 5731 de 2008 emitida por la Secretaría del Ambiente y que exige que estos no deben superar los $30 \mathrm{mg} /$ Ltr. Los valores de las fincas estudiadas se ubicaron en rangos comprendidos entre 8 y 19 $\mathrm{mg} /$ Litro (ver gráfico 8). 


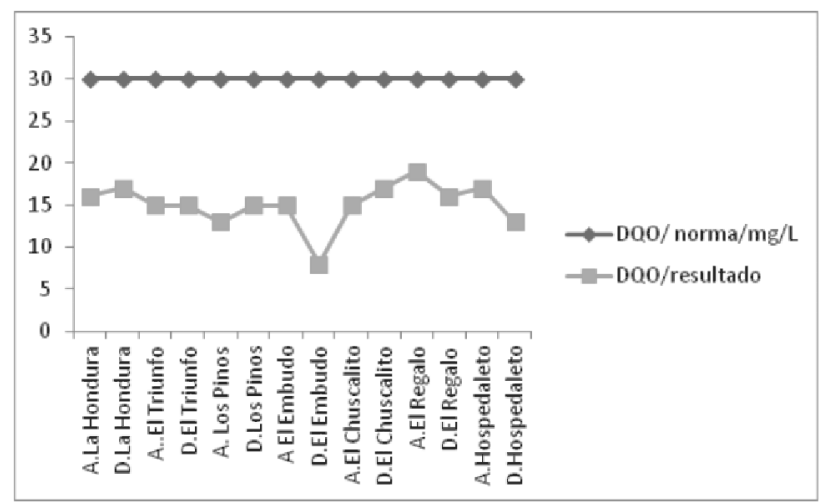

Gráfico 8. Variación de Demanda química de oxígeno mg/l en muestras de agua Superficial en la vereda Verjón Alto. Bogotá. A: Antes del sistema de producción porcino y D: Después del sistema de producción porcina

El comportamiento general de las muestras analizadas para fósforo total es típico de aguas naturales con baja afectación. Las concentraciones más elevadas se presentaron en la finca el Embudo con valores de 0.11 y $0.14 \mathrm{mg} / \mathrm{Litro}$ a la entrada y salida, respectivamente;, el valor establecido en el Acuerdo 43 de 2006 CAR para este parámetro es de $0.1 \mathrm{mg} /$ Litro. Se aplicó prueba de Dunnet para verificar si la diferencia es significativa y se encontró que estadísticamente no lo es (ver gráfico 9).

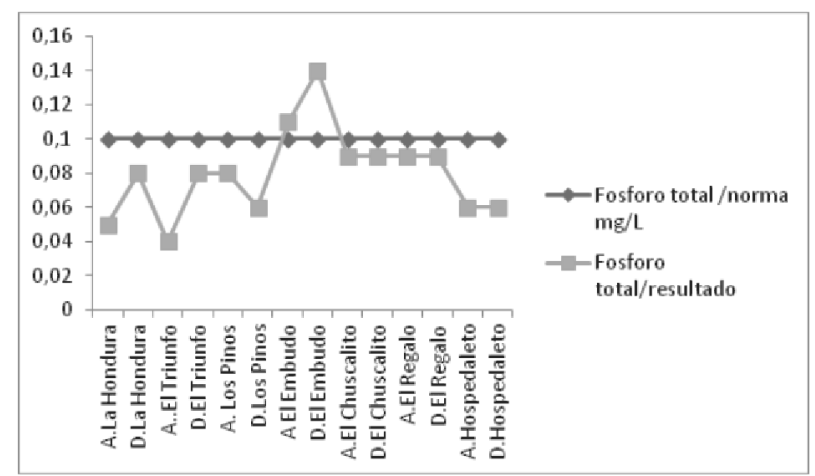

Gráfico 9. Variación de fósforo total (mg/l) en muestras de agua superficial en la vereda Verjón Alto. Bogotá. A: Antes del sistema de producción porcino y D: Después del sistema de producción porcina 
Como podía esperarse, en un área rural con restricciones de uso, inexistencia de industria y actividad agropecuaria limitada por sus condiciones de reserva hídrica, la presencia de grasas y aceites, fue mínimo y con leves variaciones, de 6 a $7 \mathrm{mg} /$ Litro. La norma exige un máximo de $10 \mathrm{mg} /$ Litro, por lo que se puede concluir que en este parámetro las condiciones del agua son óptimas (ver gráfico 10).

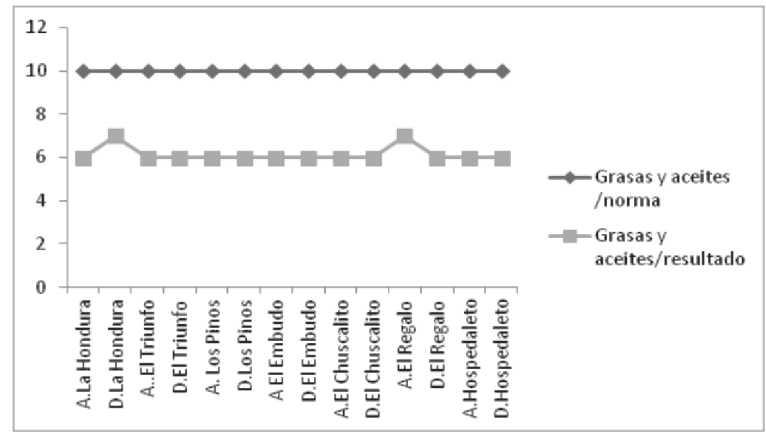

Gráfico 10. Variación de grasas y aceites $(\mathrm{mg} / \mathrm{l})$ en muestras de agua superficial en la vereda Verjón Alto. Bogotá. A: Antes del sistema de producción porcino y D: Después del sistema de producción porcina

En el nitrógeno se obtuvieron valores constantes en todas las muestras, con $0.56 \mathrm{mg} /$ litro, bastante inferior a lo estipulado por la Resolución 5731 de 2008 de la Secretaría del Ambiente que es de $1.5 \mathrm{mg} /$ Litro. Se presume que la ausencia de variación en los valores de nitrógeno entre las fincas se debe al método titulométrico utilizado para su determinación, el cual no es sensible a pequeños cambios en los contenidos de nitrógeno (ver gráfico 11).

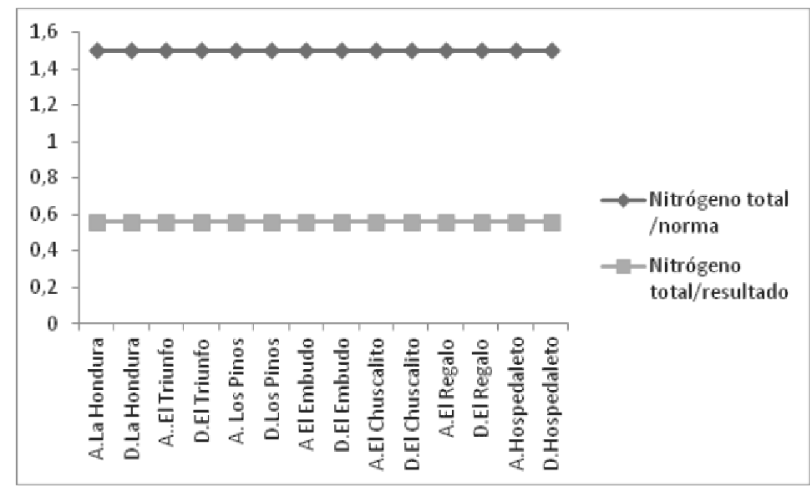

Gráfico 11. Variación de nitrógeno ( $\mathrm{mg} / \mathrm{l})$ en muestras de agua superficial en la vereda Verjón Alto. Bogotá. A: Antes del sistema de producción porcino

y D: Después del sistema de producción porcina 
La cantidad de sólidos suspendidos totales es coherente con la categoría de protección hídrica que tiene el área en estudio y su restringido uso agropecuario; por ello, encontramos valores entre 5 y $12 \mathrm{mg} /$ litro, siendo los permisibles de $10 \mathrm{mg} /$ Litro. A este parámetro se le aplicó la prueba Dunnet para establecer la diferencia entre las medias de las muestras analizadas y la norma, y se encontró que no hay diferencia significativa entre estos valores (ver gráfico 12).

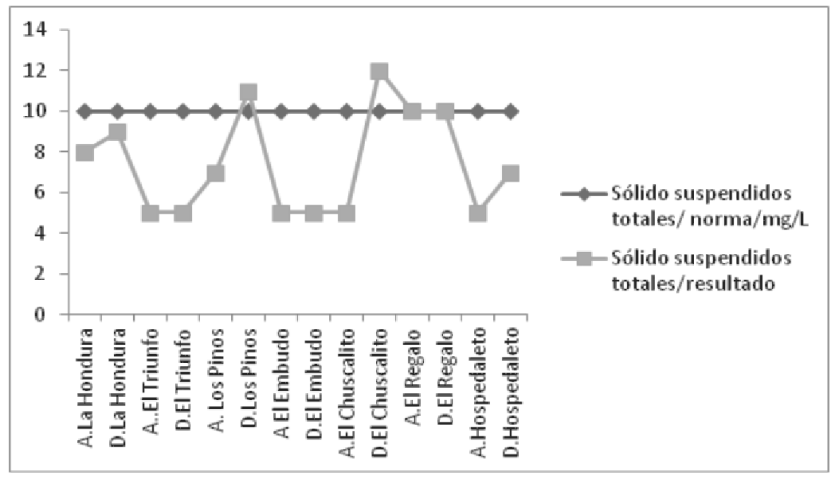

Gráfico 12. Variación sólidos suspendidos totales $(\mathrm{mg} / \mathrm{l})$ en muestras de agua superficial en la vereda Verjón Alto. Bogotá. A: Antes del sistema de producción porcino y D:después del sistema de producción

Los sólidos sedimentables presentaron valores constantes en todas las fincas y se ubicaron en $0.1 \mathrm{ml} /$ litro, estando dentro de los límites permisibles por el Acuerdo 047 de 2006 de la CAR que establece valores de $10 \mathrm{ml} /$ litro (ver gráfico 13).

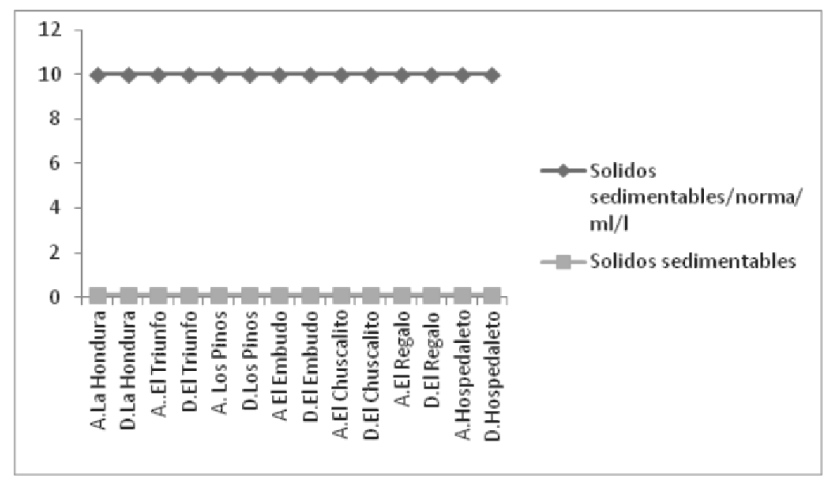

Gráfico 13. Variación de sólidos sedimentables (ml/1) en muestras de agua superficial en la Vereda Verjón Alto. Bogotá. A: Antes del sistema de producción porcino y D:

Después del sistema de producción porcina 


\section{Análisis estadístico}

Aplicando la prueba de Dunnet para el amonio, los sólidos suspendidos totales, el pH y el fósforo, se encontró que el valor crítico es mayor que el estadístico de prueba, por lo cual se acepta la hipótesis nula que plantea que la media experimental es estadísticamente igual a la media dada por la norma, con un nivel de confianza del $95 \%$.

En coliformes fecales y totales se aplicó también la prueba Dunnet y se encontró que el valor estadístico de prueba calculado es mayor que el valor crítico; por tanto, se rechaza la hipótesis nula que establece que la media experimental es igual a la media de la norma, concluyendo, con un nivel de confianza del $95 \%$, que la media experimental y la media dada por la norma son estadísticamente diferentes.

Por lo anterior, a estos resultados, en coliformes fecales y totales, se les aplicó prueba de Dunnet unilateral y se encontró que con un nivel de significancia del 95\%, el valor experimental es menor que el valor de la norma, lo cual significa que se están cumpliendo con los estándares de la norma.

A esta variable se le aplicó la prueba Dunnet unilateral y se encontró que el estadístico de prueba es mayor que el valor crítico; por tanto, con un nivel de confianza del $95 \%$ se rechaza la hipótesis nula, la cual indica que la cantidad de coliformes totales presentes en la región, cumple los estándares de la norma.

La temperatura, el DBO, el DQO, grasa y aceites, nitrógeno total, sólidos sedimentables, presentaron una media experimental dentro del rango que exige la norma, por lo cual no fue necesario realizar las pruebas específicas.

Para coliformes fecales, fósforo y sólidos suspendidos totales -SST- se encontraron resultados por fuera de la norma; sin embargo, al aplicar la prueba estadística no se hallan diferencias significativas. Para los coliformes fecales, la finca el Regalo superó el valor máximo permisible de $200 \mathrm{UFC} / 100 \mathrm{ml}$; el resultado antes del sistema productivo porcino -SPP- fue de $210 \mathrm{UFC} / 100 \mathrm{ml}$, mientras que después del SPP fue de $240 \mathrm{UFC} / 100 \mathrm{ml}$. Estos resultados coinciden con nivel alto de coliformes totales y fósforo, por lo cual se presume la presencia de residuos de excretas humanas procedentes de viviendas localizadas en áreas cercanas a la fuente de agua. Comparando estos datos con los obtenidos por Pérez (2001) en Piedad, Michoacán, México para granjas con cerdos en pie, entre 2082 y 17546, en el cual se obtuvieron coliformes fecales por valor de $1100 \mathrm{UFC} / 100 \mathrm{ml}$ para análisis de agua residual, los valores del estudio resultan sustancialmente menores.

En cuanto al fósforo, la finca el Embudo, arrojó, antes del SPP, un valor de 0,11 mg/l y después de éste, $0,14 \mathrm{mg} / \mathrm{l}$. Los resultados encontrados difieren de los reportados por Pérez (2001) en el estudio mencionado anteriormente, en los cuales los niveles de fósforo varían entre 34 y $2226 \mathrm{mg} /$ Litro. 
En el mismo sentido, se observa que los Sólidos Suspendidos Totales, en las fincas los Pinos y el Chuscalito, en la muestra tomada, después del SPP, superan a la norma que establece un máximo de $10 \mathrm{mg} / \mathrm{l}$; los valores de este parámetro, en dichas fincas fue de 11 y 12 $\mathrm{mg} / \mathrm{l}$, respectivamente. Al aplicar la prueba estadística se comprobó que la diferencia no es significativa. En la investigación realizada por Pérez (2001), se encuentran los SST entre 83 y $2020 \mathrm{mg} /$ litro, datos bastante alejados de los encontrados en las fincas objeto de estudio.

El parámetro DBO fue en todos los casos constante con valor de $2 \mathrm{mg} / \mathrm{l}$ e inferior a la norma que establece un máximo de $7 \mathrm{mg} / \mathrm{l}$. Este resultado muestra una gran diferencia con el que obtuvo Pérez (2001): un DBO entre 580 y 85802 mg/litro para análisis de agua residual.

Así mismo, el nitrógeno presentó valores constantes en todas las fincas con resultados de $0,56 \mathrm{mg} / \mathrm{l}$ e inferiores a la norma, la cual es de $1,5 \mathrm{mg} / 1$. Al respecto, el trabajo reportado por Pérez (2001), presenta rangos entre $294 \mathrm{mg} / \mathrm{Litro}$ y $1520 \mathrm{mg} / \mathrm{litro}$, muy distantes de los presentados en el sitio de estudio.

Los sólidos sedimentables tampoco tuvieron variación entre las diferentes fincas con valores de $0,1 \mathrm{mg} / 1$ y por debajo de la norma que establece $10 \mathrm{mg} / 1$. Estos datos están cercanos a los reportados por Pérez (2001), los cuales se encuentran entre 0.2 y $5.0 \mathrm{mg} /$ litro, de acuerdo con datos tomados por porcicultores.

\section{CONCLUSIONES Y RECOMENDACIONES}

Las medidas denominadas "Cero vertimientos" adoptadas por los productores de cerdo en la reserva forestal de los cerros orientales de Bogotá, vereda el Verjón alto, área rural de Bogotá, localizada sobre los 3000-3200 m.s.n.m., para mitigar los efectos producidos por los residuos de unidades productivas en aguas superficiales han sido efectivas, por las siguientes razones:

El parámetro físico de temperatura, no evidenció variaciones significativas respecto a la normatividad consultada y su valor de 10 grados centígrados se mantuvo constante en todas las muestras analizadas y es coherente con las condiciones climáticas de la región.

Los parámetros químicos, oxígeno disuelto, demanda bioquímica de oxígeno (DBO), demanda química de oxígeno (DQO), grasas y aceites, nitrógeno total y sólidos sedimentables, presentaron valores dentro de los límites permisibles, por lo cual no fue necesario realizar pruebas estadísticas adicionales y sus valores expresan la alta calidad del agua de la zona estudiada, como resultado de las medidas adoptadas por los productores de porcinos y el limitado número de cabezas por unidad productiva.

Los parámetros químicos, $\mathrm{pH}$, fósforo total, sólidos suspendidos totales, amonio, presentaron en algunas fincas valores por fuera de la norma consultada; al aplicarse las pruebas estadísticas se concluyó que las diferencias no eran significativas. 
Los parámetros microbiológicos, coliformes fecales y coliformes totales mostraron para la finca el Regalo valores superiores a la norma, por lo cual se aplicaron pruebas estadísticas y se concluyó que el conjunto de muestras del agua de la región no presentaba contaminación, en relación con la de la norma.

Se puede establecer que el agua de la región en estudio es de alta calidad, en función del uso que se le proyecte dar, como es: consumo humano con tratamiento convencional, fauna y flora, uso agrícola y uso pecuario. Sin embargo, se recomienda hacer un estudio más específico en la finca el Regalo, ya que los valores de coliformes totales exceden lo establecido por la norma.

Es conveniente que el Decreto 1594 de 1984, que regula en el ámbito nacional los usos y establece algunos criterios sobre calidad de agua se revise, con el fin de precisar para cada parámetro (físico, químico y microbiológico) valores permisibles. Lo anterior, con la finalidad de facilitar criterios unificados para la toma de decisiones en el control y seguimiento a la calidad hídrica afectada por actividades productivas.

Se recomienda adelantar estudios en la misma zona, en los cuales se comparen los resultados obtenidos en la calidad de agua procedente de fincas que apliquen el programa cero vertimientos con fincas en las cuales no se implemente este programa. Igualmente, es necesario verificar la posible afectación del agua subterránea por los sistemas productivos, actualmente desarrollados en la vereda Verjón Alto del área rural de Bogotá.

Se recomienda, realizar una nueva prueba del contenido de amonio en el agua superficial de las fincas Hondura y Pinos para verificar si existen "datos raros" que permitan confirmar los resultados arrojados en el laboratorio o identificar situaciones que estén generando altos índices de esta sustancia, diferentes al sistema de producción porcina.

\section{AGRADECIMIENTOS}

A Priscila Rey Vásquez, decana de la Escuela de Ciencias Agrícolas, Pecuarias y del Medio Ambiente de la Universidad Nacional Abierta y a Distancia por el apoyo financiero para la ejecución de este proyecto. A Julio Ignacio García, Ingeniero Químico con Maestría en Estadística, quien apoyó el trabajo estadístico.

\section{REFERENCIAS BIBLIOGRÁFICAS}

ALCALDÍA MAYOR DE BOGOTÁ D.C. 2007. Secretaría Distrital del Ambiente y Empresa de Acueducto y Alcantarillado de Bogotá. Calidad de sistemas hídricos de Bogotá. 299 p.

BRAVO- ALCÁNTARA, A. 2008. Evaluación del suministro de agua residual tratada por separación-sedimentación-filtración en la salud de cerdos destetados. Revista Técnica pecuaria en México. Vol. 46 (3) 287-302. 
CAMARGO, G. \& SALAMANCA, B. 2.000. Protocolo Distrital de Restauración Ecológica. 288 p.

CASTRILlÓN, Q., O., JiMÉNEZ P. A. \& BEDOYA M., O. 2004. Porquinaza en la alimentación animal. Revista Lasallista de investigación, Antioquia Colombia Vol. 1 (1) $72-76$.

CUBILlOS, A. 1978. Parámetros y características de las aguas residuales. Proyecto de desarrollo tecnológico de las instituciones de abastecimiento de agua potable y alcantarillado -DTIAPA- División de Protección de la salud ambiental. Centro panamericano de ingeniería sanitaria y ciencias del ambiente -CEPIS-. Lima, Perú. 31 pág. Disponible en internet: http://bvsde.per.paho.org/bvsacd/scan2/011643/011643-09.pdf.

CORPORACIÓNAUTÓNOMA REGIONAL DE CUNDINAMARCA, Cámara de Comercio de Bogotá y Asociación Colombiana de Porcicultores. 2006. Programa de diagnóstico y acompañamiento productores porcícolas cerros orientales. Asistencia Técnica para la implementación de cero vertimientos y manejo apropiado de residuo,.pp25-28.

CORPORACIÓN AUTÓNOMA REGIONAL DE CUNDINAMARCA -CAR- 2006. Acuerdo CAR 043 de 2006 "por el cual se establecen objetivos de calidad de agua para la Cuenca del Río Bogotá a lograr en el 2020”, para valores asignados a embalses.

ESPINAL, C. F., MARTÍNEZ, H. \& ACEVEDO, X. 2005. La cadena de cereales, alimentos balanceados para animales, avicultura y porcicultura en Colombia una mirada global de su estructura y dinámica 1991-2005 Ministerio de Agricultura y Desarrollo Rural. Observatorio Agro cadenas Colombia. Documento de trabajo No. 51. Marzo. 56 p. disponible en la web. http://www.agronet.gov.co/www/docs_agronet/200511215360_ caracterizacion_cereales.pdf.

INSTITUTO DE HIDROLOGÍA, Meteorología y Estudios Ambientales -IDEAM- 2001. "Medio ambiente en Colombia". Editor Pablo Leyva. Segunda edición.

LÓPEZ, F. \& PINZÓN, M. 1998. CORPOICA-DAMA: Diagnóstico Agropecuario Integrado de las Localidades de Ciudad Bolívar, Chapinero, Santa Fe, Sumapaz y Usme en el D.C.

PÉREZ-ESPEJO, R. 2006. Granjas porcinas y medio ambiente: Contaminación del agua en La Piedad Michoacán. UNAM, SEMARNAT, P y V, México, D.F. 194 pág. Disponible en internet: http://books.google.com.co/books?hl=es\&lr=\&id=7ciPXwEu 2b4C\&oi=fnd\&pg $=$ PA9 \&dq $=$ agua + residual + de + origen + porcino $+\&$ ots $=$ OUFAfqb05\&sig=XI8tG5OQD5dTp4ua_O0IOL4uhIk\#PPA7,M1. 
PICCININI, A. 1995. Eliminación de las aguas residuales porcinas: aspectos económicos y ambientales (I). Mundo ganadero: cuaderno porcino No 5 Mayo, 1995 pp: 50-55. Disponible en internet. http://www.mapa.es/ministerio/pags/biblioteca/revistas/pdf_MG/ MG_1995_5_95_50_55.pdf.

SECRETARIA DEL AMBIENTE DE BOGOTÁ. 2008. Resolución 5731 de 2008 Objetivos de calidad de agua a 10 años (2009-2019) para los ríos tributarios del río Bogotá: Tunjuelito, canal Torca río Fucha y Salitre. Se tomó parámetro de primer tramo (entrada a perímetro urbano) de río Tunjuelito.

YOUNG, J., CLESCERI, L. \&. KAMHAWY S. 2005. Changes in the biochemical oxygen demand procedure in the 21 st edition of Standard Methods for the Examination of Water and Wastewater. Water environment research Vol 77 (4) 404-410. 